$\$$ Research Square
Preprints are preliminary reports that have not undergone peer review.

They should not be considered conclusive, used to inform clinical practice, or referenced by the media as validated information.

\title{
The Factors Influencing the Implementation of Clinical Guidelines in the World; a Systematic Review Protocol
}

Javad Babaei

Tabriz Medical University: Tabriz University of Medical Sciences

Sakineh Hajebrahimi

Tabriz Medical University: Tabriz University of Medical Sciences

Ali Jannati

Tabriz Medical University: Tabriz University of Medical Sciences

Mina Azizzadeh ( $\sim$ azizzadehmina@gmail.com )

Tabriz Medical University: Tabriz University of Medical Sciences https://orcid.org/0000-0002-7887-0351

Protocol

Keywords: Factors, Barriers, Implementation, clinical guidelines

Posted Date: January 15th, 2021

DOI: https://doi.org/10.21203/rs.3.rs-144665/v1

License: (c) (i) This work is licensed under a Creative Commons Attribution 4.0 International License. Read Full License 


\section{Abstract}

Background: Clinical guidelines are a systematic collection of the latest and most authoritative scientific evidence that describes thediagnostic steps and methods of clinical treatment of a patient in a categorized manner, taking into account priorities, effectiveness, and cost-effectiveness. It is necessary to explore and categorize influencing factors in the implementation of clinical guidelines worldwide to pave the way for their implementation in medical centers.

Methods: In this systematic review we will search Scopus, PubMed, Web of sciences, clinical key, Google Scholar (Search Engine as well as Iranian data bases including: Magiran, SID, Irandoc, Iranmedex. We will include all related original studies that generally cover all relevant outcomes to determine the factors influencing the success of the implementation of clinical guidelines and to identify barriers and facilitators as well.The quality of the included studies will be assessed by appropriate Joanna Briggs Institute (JBI) Checklists according to the type of studies. The selection and appraisal process will be performed by two members of the research team; and in case of disagreement between the two researchers, the third person will review the articles.

Discussion: Identifying the facilitators and factors affecting the implementation of clinical guidelines as well as the related obstacles is expected to pave the way for helping managers, policymakers and health planners in implementing them correctly.

\section{Systematic review registration: CRD42020201350}

\section{Background}

Clinical guidelines are an important and objective part of physicians' evidence-based practice for decision making. Carefully formulated clinical guidelines are the result of a combination of relevant research linking evidence to clinical practice(1). Clinical guidelines make the sophisticated scientific texts available to easy-to-use health care providers(2). Clinical guidelines are a systematic collection of the latest and most authoritative scientific evidence that describes the methods of clinical treatment of a patient in a categorized manner taking into account the priorities, effectiveness, and cost-effectiveness(3).

Clinical guidelines are developed to facilitate physicians' decisions about patients' medical care in specific medical situations $(4,5)$. In recent years, clinical guidelines have been used in world health systems to address some of the problems in the health system. Among the purposes of using clinical guidelines are the following: Providing an appropriate service model based on the best available evidence and documentation and in limited cases, general consensus, providing a basis and rational model for referring patients, reducing the differences of opinion in clinical practice, providing focus on continuing education and quality control processes including clinical auditing, focus on weaknesses in existing documentation and points that need to be focused on for future researches, advancing, and promoting efficient use of Resources, control of costly treatment and diagnostic applications, and providing evidence-based context for insurance tariffs $(6,7)$.

Clinical guidelines assist physicians and nurses in providing the best clinical guidance. Furthermore, changing in the process of services provided by following these guidelines will lead to cost savings; Therefore, paying attention to clinical guidelines is a predominant part of clinical governance that assists physicians and nurses in developing, following up, monitoring and improving standards $(8,9)$. 
On the other hand, the implementation of clinical guidelines has not been easy and sometimes, it has encountered obstacles(10). According to research, clinical guidelines are not usually used. To help implement clinical guidelines, obstacles must be identified and the right strategies must be considered for their successful implementation (1113). Among these problems and obstacles there can be; Organizational constraints, ambiguous and complex guidelines and their incompleteness, disagreement with the guidelines due to non-compliance with the executive environment and lack of sufficient evidence, knowledge, and attitude, inefficiency and previous inefficiency, lack of attention to patients' priorities and wishes, lack of the possibility of updating for use, and environmental factors, lack of time management and time constraints, etc(10).

Among the existing studies, lack of time required to use clinical guidelines and lack of knowledge and understanding about them have been introduced as the most common obstacles in the implementation of clinical guidelines(14-17).

The results of a review of the factors influencing the implementation of clinical guidelines show that the use of multiple factors is more effective than the use of a single factor to succeed in this field. In general, the factors influencing the successful implementation of clinical guidelines are: characteristics of clinical guidelines, including their fluency and comprehensibility, characteristics of specialists such as familiarity with clinical guidelines and their content, patient characteristics such as having two diseases simultaneously. Finally, environmental characteristics such as adequate attention and time of employees and the support of colleagues and supervisors in the workplace are considered as the important factors (18).

In a study conducted in China entitled "Obstacles to the implementation of clinical guidelines", the results of the study showed that the main obstacles are related to the inherent defects in the clinical guidelines themselves and the existence of defects and ambiguities in the functioning of the system. More precisely, the main obstacles were: lack of ease of use of clinical guidelines, for example, in terms of unrelatedness to the hospital information system, lack of evidence from Chinese samples (meaning to be native), usability problems such as lack of transparency and feasibility in action (19).

In other study findings, the most important obstacles to full implementation of clinical guidelines include; Lack of resources, lack of knowledge of the content of the guidelines, non-acceptance of the recommendations of the clinical guidelines and the failure of the clinical guidelines to make a clear (10).

The results of another study of barriers to the use of clinical guidelines among physicians in the Netherlands showed that Dutch general practitioners have a positive attitude towards clinical guidelines. This study reports the high level of adherence and low level of barriers among physicians. In this study, most of the perceived barriers are about the patient-related recommendations and this indicates that current guidelines do not always adequately address patients' preferences, needs, and abilities(20). The importance of patients' opinions in drafting clinical guidelines for improving treatment outcomes has been emphasized(21).

In order to pave the way for the implementation of clinical guidelines in medical centers to achieve beneficial clinical benefits for physicians and patients and to improve the quality of clinical services and to achieve the economic benefits arising from it, it is necessary to explore and categorize the influencing factors in the implementation of clinical guidelines throughout the world. The specific objectives of this review are:

1-Identifying the factors affecting and facilitating the implementation of clinical guidelines in medical centers in the world 
2- Identifying the obstacles and limitations of implementing clinical guidelines in medical centers in the world

\section{Methods/design}

\section{Study method}

We designed this protocol for a systematic review based on the Preferred Reporting Items for Systematic Reviews and Meta-Analyses Protocols (PRISMA-P) guidelines(22). This protocol has been registered in PROSPERO (registration number CRD42020201350).

\section{Research questions}

1. What are the factors that facilitate the implementation of clinical guidelines in medical centers in the world?

2. What factors hinder the implementation of clinical guidelines in medical centers around the world?

\section{Eligibility criteria}

We will include all relevant quantitative or qualitative original studies to determine the factors influencing the success of clinical guidelines and to identify barriers and facilitators. Effective agents and facilitators are those measures that have been taken in recent years to pave the way for the implementation of clinical guidelines in medical centers around the world. It is also important to identify the barriers that have limited the implementation of the guidelines. The criteria for the study eligibility in this review including population, context, and outcome are described below.

\section{Population}

All hospitals or third level health care centers that have identified and reported on effective factors or significant barriers to the successful implementation of clinical guidelines.

\section{Intervention, exposures}

This study attempts to find the factors affecting the implementation of clinical guidelines by physicians in hospitals to help their successful implementation to achieve the goals of clinical guidelines in promoting health. Factors influencing the implementation of clinical guidelines are those factors that help to implement clinical guidelines correctly and successfully by influencing therapeutic and non-therapeutic components and various aspects in the hospital. The factors include; Hospital professional cultures, economic factors, factors related to insurance, how clinical guidelines are set up, and so on.

\section{Context}

The context of the study includes all general and specialized hospitals or healthcare centers in various fields of medicine in the countries worldwide that have experiences concerning the factors influencing the implementation of guidelines.

\section{Outcome}

Outcomes of this study are the effective factors to implement clinical guidelines successfully and identify the pertinent barriers to improve treatment and care outcomes in patients. 


\section{Types of studies}

We will include all related original studies that generally cover all the pertinent studies to determine the factors influencing the success of clinical guidelines and to identify the barriers and facilitators. (Most qualitative and quantitative studies include descriptive and analytical, possibly observational, and perhaps a small number of interventions, and mix method studies.) Studies that are related to primary health care centers and nursing related field will be excluded. English and Persian publications and their period from 2000 to 2020 will also be included.

\section{Search strategy}

The following databases will be searched: Scopus, PubMed, Web of sciences, clinical key,Google Scholar (SearchEngine)as well as Iranian data bases including: Magiran, SID, Irandoc, Iranmedex. For each database, the appropriate strategy will be selected. We will hand-search studies and reference lists relevant to systematic reviews. We will supplement the electronic database searches with website and grey literature searches. Moreover, we will search relevant organizations websites such as World Health Organization. We will restrict the date of publications from the $1^{\text {st }}$ of July 2020 to the 7th of July 2020.

\section{Study selection}

All found articles will be imported into Endnote software. After removing duplicate items, the titles and abstracts of articles will be reviewed based on inclusion and exclusion criteria and, if eligible, will be included in the study. The search process will be performed by two members of the research team (M-A and J-B). In case of disagreement between the two researchers, the third person will review the articles (A-J). The title and abstract of the articles are reviewed by two researchers based on the entry and exit criteria. Articles that go to the next stage after reviewing the title and abstract will be reviewed based on the full text and the full text will be screened independently by two people and the final decision will be made based on entry and exit criteria. Studies that met the inclusion criteria will be qualitatively assessed and the data from these studies will be entered in the data extraction form.

\section{Quality assessment}

The quality of the included studies will be assessed by appropriate Joanna Briggs Institute (JBI) Checklists according to the type of studies(23). JBI has separate checklists for various studies, including cross-sectional studies, case-control studies, RCT studies, quasi-experimental studies, and qualitative studies. The responses options for the checklist included "yes", "no", "unclear", or "not applicable". The risk of bias will be assessed by two members of the research team separately (M-A and S-H). In case of disagreement between the two researchers, the third person will review the articles $(A-J)$. According to the quality, studies will be reported in separate tables. The quality of studies will be graded high, medium, low: a rigorous and robust scientific approach, some flaws and medium scientific value and Low-serious flaws and poor scientific value.

\section{Data extraction}

To collect the data, a data mining table will be used which will be designed by the researchers. The required information will be extracted from the studies and recorded in Excel 2016, including the name of the first author, year of publication, geographical location of the study, duration of the study, study design, target group, experiences, implementation barriers, effective and facilitating factors, etc. These items will be selected by all of the authors (MA, J-B, A-J, and S-H-E) according to the key questions of the study. The members of the research will modified the draft of extraction form during the process. 


\section{Data synthesis}

A thematic analysis will be conducted using the data extraction table. We will follow the six stages of framework synthesis to synthetize our qualitative data. A thematic analysis is focused on examining and reporting on patterns (or 'themes') within the data, either semantically present or latent. Thematic analysis is focused on determining the different themes within a subject and their inter-relationships. Methodologically, the thematic analysis will be performed according to six-phase guide:(1) Familiarization with the data; (2) Generating initial codes; (3) Searching for themes; (4) Reviewing themes; (5) Defining and naming themes; and (6) Producing the report.

\section{Discussion}

Clinical guidelines are gaining more and more attention around the world(24). Adherence to clinical guidelines, which are the same as evidence-based standards, reduces the mortality rate and improves treatment outcomes, thereby improving the quality of life(25). Obviously, the implementation of clinical guidelines in health centers is essential. For successful implementation of clinical guidelines, it is necessary to identify the affecting facilitators and factors along with obstacles. The results of this systematic study are expected to pave the way for the implementation of guidelines successfully. Identifying the factors influencing the implementation of clinical guidelines was a great help for managers, policymakers and health planners to implement them correctly and improve health outcomes.

\section{Strengths And Limitations Of This Study}

One of the strengths of the present study is its being up-to-date in terms of time, which means that it will be one of the latest studies in this field. This is important due to the ongoing changes in the health system. Attempts to qualitatively evaluate the input studies in this review based on valid tools can be considered as other strengths of this study. These provide a credible resource for health decision makers. As a limitation of the present study, we can mention the loss of studies conducted in languages other than Persian and English which can lead to the loss of some of the recommendations for the successful implementation of clinical guidelines in medical centers.

\section{Abbreviations}

JBI: Joanna Briggs Institute; PRISMA: Preferred Reporting Items for SystematicReview and Meta-Analysis Statement; PROSPERO: International ProspectiveRegister of Systematic Reviews; SID: Scientific Information Database

\section{Declarations}

\section{Acknowledgements}

We would like to appreciate MS. Fatemeh Arabi Basharik and Dr. Ehsan Khara for their valuable advice.

\section{Authors' contributions}

A-J conceptualized the research idea. M-A initiated the protocol andwrote the manuscript. S-H-E and A-J contributed to the design of protocoland provided the critical revision of the manuscript. J-B drafted the inclusionand exclusion criteria and provided the data extraction form. J-B helped to formulate the search strategy, reviewed the whole 
manuscript,and provided substantial comments for editing. All authors critically reviewedthe study protocol and approved the final manuscript.

\section{Funding}

This protocol systematic review is a part of a Ph.D. project. Funding isprovided by the School of Management and Medical Informatics, TabrizUniversity of Medical Sciences, Tabriz, Iran. The funder had no involvementin the study design, the writing of the manuscript, or the submission forpublication.

\section{Availability of data and materials}

Not applicable

\section{Ethics approval and consent to participate}

Not applicable

\section{Consent for publication}

Not applicable

\section{Competing interests}

The authors declare that they have no competing interests.

\section{References}

1. Andrews JC, Schünemann HJ, Oxman AD, Pottie K, Meerpohl JJ, Coello PA, et al. GRADE guidelines: 15. Going from evidence to recommendation-determinants of a recommendation's direction and strength. Journal of clinical epidemiology. 2013;66(7):726-35.

2. Graham R, Mancher M, Wolman D, Greenfield S, Steinberg E. Institute of Medicine. Clinical practice guidelines we can trust. Washington, DC: The National Academies Press; 2011.

3. The meaning of Gideline. Evidence Based Research Center: Tabriz Medical Sciences Evidence Based Research Center; Available from:

https://ircebm.tbzmed.ac.ir/Page/108/\%DA\%AF\%D8\%A7\%DB\%8C\%D8\%AF\%D9\%84\%D8\%A7\%DB\%8C\%D9\%86\%DA\%86\%DB\%8C\%D8\%B3\%D8\%AA\%D8\%9F.html.

4. Field MJ, Lohr KN. A provisional instrument for assessing clinical practice guidelines. Guidelines for clinical practice: From development to use: National Academies Press (US); 1992.

5. Dabbagh A, Ghasemi M, Zolfaghari B, Jamshidi H. A revision to the professional roles of pharmacists in the national health system: the attitudes of.

6. Rhinehart E, Goldmann D, O'Rourke E. Adaptation of the Centers for Disease Control guidelines for the prevention of nosocomial infection in a pediatric intensive care unit in Jakarta, Indonesia. The American journal of medicine. 1991;91(3):S213-S20.

7. Merritt TA, Palmer D, Bergman DA, Shiono PH. Clinical practice guidelines in pediatric and newborn medicine: implications for their use in practice. Pediatrics. 1997;99(1):100-14. 
8. Eccles MP, Grimshaw JM, Shekelle P, Schünemann HJ, Woolf S. Developing clinical practice guidelines: target audiences, identifying topics for guidelines, guideline group composition and functioning and conflicts of interest. Implementation science. 2012;7(1):60.

9. Guyatt G, Rennie D, Meade M, Cook D. Users' guides to the medical literature: a manual for evidence-based clinical practice: AMA press Chicago; 2002.

10. Clark M. Barriers to the implementation of clinical guidelines. Journal of Tissue Viability. 2003;13(2):62-72.

11. Fischer F, Lange K, Klose K, Greiner W, Kraemer A, editors. Barriers and strategies in guideline implementation-a scoping review. Healthcare; 2016: Multidisciplinary Digital Publishing Institute.

12. Grol R, Grimshaw J. From best evidence to best practice: effective implementation of change in patients' care. The lancet. 2003;362(9391):1225-30.

13. Grol R, Wensing M. What drives change? Barriers to and incentives for achieving evidence-based practice. Medical Journal of Australia. 2004;180:S57-S60.

14. Brassard E, Archambault P, Lacombe G, St-Onge M. Barriers and Facilitators of Intensivists' Adherence to Hyperinsulinemia-Euglycemia Therapy in the Treatment of Calcium Channel Blocker Poisoning. Journal of Medical Toxicology. 2018;14(4):283-94.

15. Curtis K, Van C, Lam M, Asha S, Unsworth A, Clements A, et al. Implementation evaluation and refinement of an intervention to improve blunt chest injury management-A mixed-methods study. Journal of clinical nursing. 2017;26(23-24):4506-18.

16. Newlands R, Duncan EM, Prior M, Elouafkaoui P, Elders A, Young L, et al. Barriers and facilitators of evidencebased management of patients with bacterial infections among general dental practitioners: a theory-informed interview study. Implementation science. 2015;11(1):11.

17. McBain H, Lamontagne-Godwin F, Haddad M, Simpson A, Chapman J, Jones J, et al. Implementation of Type 2 diabetes guidelines in people with severe mental illness (SMI). Diabetic Medicine. 2018;35(S1):P518.

18. Francke AL, Smit MC, de Veer AJ, Mistiaen P. Factors influencing the implementation of clinical guidelines for health care professionals: a systematic meta-review. BMC medical informatics and decision making. 2008;8(1):38.

19. Jin Y, Li Z, Han F, Huang D, Huang Q, Cao Y, et al. Barriers and enablers for the implementation of clinical practice guidelines in China: a mixed-method study. BMJ open. 2019;9(9):e026328.

20. Lugtenberg M, Burgers JS, Besters CF, Han D, Westert GP. Perceived barriers to guideline adherence: a survey among general practitioners. BMC family practice. 2011;12(1):98.

21. Lingner H, Burger B, Kardos $P$, Criee $C$, Worth H, Hummers-Pradier E. What patients really think about asthma guidelines: barriers to guideline implementation from the patients' perspective. BMC pulmonary medicine. 2017;17(1):1-12.

22. Liberati A, Altman DG, Tetzlaff J, Mulrow C, Gøtzsche PC, loannidis JP, et al. The PRISMA statement for reporting systematic reviews and meta-analyses of studies that evaluate health care interventions: explanation and elaboration. Journal of clinical epidemiology. 2009;62(10):e1-e34.

23. Institute JBI. Critical appraisal tools. Available from: https://joannabriggs.org/ebp/critical_appraisal_tools.

24. Garner P, Kale R, Dickson R, Dans T, Salinas R. Implementing research findings in developing countries. Bmj. 1998;317(7157):531-5.

25. Yoo B-S, Oh J, Hong B-K, Shin D-H, Bae J-H, Yang DH, et al. SUrvey of Guideline Adherence for Treatment of Systolic Heart Failure in Real World (SUGAR): a multi-center, retrospective, observational study. PloS one. 
2014;9(1):e86596.

\section{Supplementary Files}

This is a list of supplementary files associated with this preprint. Click to download.

- FUNDAPPROVALCERTIFICATE.pdf

- PRISMAP2015checklist.docx

- researchethicscertificate.pdf

- searchstrategyexample.docx 